Background and Aim Acute renal failure occasionally occurs in neonates secondary to generalized sepsis or major cardiac surgery. Insertion of a peritoneal dilyasis (PD) catheter is needed in majority of cases. Open laparotomy techniques are prone to bleeding and dialysate leakage. Percutaneous bed-side insertion of PDs is the preferred method in our setting.

Methods In a retrospective study, over a 8-year period, neonatal PDs were inserted using Palmer's point at the bed-side in intensive care unit. Plamer's point is an anatomical landmark; it is on the left anterior axillary line and just in front of the $10^{\text {th }}$ rib. This point is known to have the least amount of adhesions and therefore blind insertion of catheters and trocars are least likely to cause iatrogenic gut perforations.

Results 51 PDs were inserted in that period; in 7 cases pervious gastrostomy or pacemaker forced an insertion of $\mathrm{PD}$ in the right hypochondrium resulting in 2 minor liver injuries. The remaining 44 PDs using Palmer's point were successfully placed. Dialysis was carried out for 2 weeks on average and was successful in controlling renal failure in all cases. However, in the long-term, 12 patients succumb to their septic, respiratory or cardiac lesions subsequently.

Conclusion Percutaneous bed-side insertion of PD catheter in neonates is possible, safe and successful using Palmer's point.

\section{TRANSFORMING GROWTH FACTOR-B1 IN CHILDREN WITH CHRONIC LIVER DISEASE}

doi:10.1136/archdischild-2012-302724.0797

H Soliman. Banha University Hospital, Banha, Egypt

Background Chronic liver disease is marked by the gradual destruction of liver tissue over time. Hepatic fibrosis is a wound healing response ending eventually with cirrhosis. Transforming growth factor beta among the cytokines and growth factors known to influence lipocyte collagen synthesis.

Objectives The aim was to study the correlation between serum TGF- $\beta 1$ and liver fibrosis and dysfunction.

Patients and methods: This retrospective descriptive study was carried out in the Pediatric Hepatology Clinic, Benha University Hospital, to review the files of patients diagnosed with chronic liver disease during the period from June 2008 to January 2009 and they were 40 cases. They were classified to; group IA (minimal or no liver fibrosis), group IB (marked liver fibrosis), 10 normal children (group II, control group) were collected matching with patients in sex and age.

Result Using METAVIR score, the mean TGFbeta1 in group II was higher than in group I, and the difference was highly significant between group IA and group II. Moreover the difference was highly significant between group IB and group II, but no significant difference between group IA and group III. The mean TGF beta is highly significant $(p<0.01)$ between child-Pugh class $A$ and $C$, also between Child-Pugh class A and B, but no significant difference $(p>0.05)$ between Child-Pugh class B and C.

Conclusion TGF- $\beta-1$ cytokine increases in the plasma of children with chronic liver diseases of various etiology and may be correlated to the chronicity of the liver disease and the extent of the hepatic injury.

\section{ELEVATED VALUES OF SERUM TRANSAMINASES IN CHILDREN-ONE YEAR EPIDEMIOLOGY AND ETIOLOGY STUDY}

doi:10.1136/archdischild-2012-302724.0798

D Pacurar, I Simion, R Nicolaescu. 'Grigore Alexandrescu' Children's Hospital, Bucharest, Romania

Aminotransferases are used worldwide for the screening of liver and muscular diseases.
Purpose To indicate the prevalence of elevated serum aminotransferases at the time of child's admission, the epidemiologic aspects of these abnormal values.

Materials and Methods We performed an observational, retrospective study (January-December 2008) in which we studied demographic data (age, sex distrbution), biological findings, correlation between age and level of enzymes or etiology. The analysis was performed usig Microsoft Excel 2007 and SPSS Statistics 17.0.

Results We studied 925 children aged 1 month-18 years (8\% of 11797 admission in a pediatric hospital) with abnormal serum aminotransferases. The highest frequency was noticed in male $(54.4 \%$, $p<0,008)$. In the majority of cases hepatocytolysis was minor. Corelating the aminotransferases values with age we discovered that lower values are more prevalent with smaller ages, while higher values were encountered in children above 14 years. As etiology the majority of cases is represented by cytolysis with no obvious cause $(87 \%)$ and, out of this population, by non-specific infectious diseases.

Conclusions Elevated serum aminotransferases are frequently encountered in hospitalized children. The lower values prevail in the context of non-specific infectious diseases. Small children are more susceptible to hepato-muscular injury by non-specific infectious diseases. Therefore we highlight the necessity for further prospective studies in order to investigate if incidentally discovered abnormal serum aminotransferaseses children.

\section{FACTORS INFLUENCING EARLY NEONATAL MORTALITY IN RETRIEVED EXTREME PRETERM NEONATES}

doi:10.1136/archdischild-2012-302724.0799

'K Wilson, ${ }^{1} \mathrm{~A}$ Nagy, ${ }^{1} \mathrm{C}$ Green, ${ }^{2} \mathrm{D}$ Boyd, ${ }^{2} \mathrm{~N}$ Ratnavel, ${ }^{2} \mathrm{~S}$ Mohinuddin. ${ }^{1}$ Barts and the London School of Medicine and Dentistry; ${ }^{2}$ London Neonatal Transfer Service, Barts Health NHS Trust, London, UK

Background and Aims Neonatal care is increasingly delivered within regionalised networks, often necessitating transfer of vulnerable preterm babies from local neonatal units to neonatal intensive care units (NICU). Extreme preterm infants (gestation $<28$ weeks) born in hospitals without a NICU have a relatively higher mortality rate than those inborn in hospitals with NICU. In this study we aim to investigate the factors that impact on early (7-day) neonatal mortality in retrieved extreme preterm infants.

Methods Inclusion criteria $(<28$ weeks gestation, transfer $<24$ hours of birth, complete data entry) were applied to all entries in a regional transfer service database between January 2005 and December 2011 ( $n=7669)$ leaving 621. Early mortality was analysed against gestational age, birth weight, lowest $\mathrm{pH}$, temperatures on NTS arrival at referring unit (T1), departure from referring unit (T2) and arrival at the receiving unit (T3). Statistical analysis was carried out using SPSS v18.

Results 7-day mortality was 88 (14.17\%). Mean (Range) for gestational age was 25.35 weeks (22.0-27.86), birth weight 794g (440$1650)$ and lowest $\mathrm{pH}$ (prior to transfer) was 7.28 (6.90-7.53). Only gestational age $(<0.001)$, birth weight $(\mathrm{p}<0.001)$ and lowest $\mathrm{pH}$ affected mortality individually $(\mathrm{p}<0.001)$. Mortality was not significantly affected by T1 $(p=0.152), T 2(p=0.265)$ and T3 $(p=0.065)$. To control for confounding, we performed logistic regression, after which gestational age $(\mathrm{p}<0.001)$ and lowest $\mathrm{pH}(\mathrm{p}=0.001)$ remained significant.

Conclusion Gestational age and lowest $\mathrm{pH}$ significantly influence 7-day mortality within retrieved extreme preterm infants.

800 ETIOLOGY AND OUTCOME OF HYDROPS FETALIS: A SINGLE CENTER EXPERIENCE IN TURKEY

doi:10.1136/archdischild-2012-302724.0800 
S Takci, M Gharibzadeh, M Yurdakok, Z Akcoren, A Korkmaz, S Yigit. Hacettepe University Ihsan Dogramaci Childrens' Hospital, Ankara, Turkey

Objective We aimed to define the etiologic features, outcome of liveborn hydrops fetalis and identify predictors of mortality in a single tertiary unit over an 11-year period.

Method Medical records of liveborn neonates with hydrops fetalis between 2000-2011 were reviewed retrospectively. Demographic data, antenatal interventions clinical manifestations, laboratory findings, outcomes and complete or partial autopsy records were analyzed.

Results Twenty six live born cases of hydrops fetalis with a sex distribution of 33 males and 29 females from the 16,200 live born deliveries in our hospital; an incidence of 3.8/1000 live births. 28 infants $(45 \%)$ were diagnosed as immune hydrops fetalis. Overall, 32 infants (50\%) survived to discharge. The survival rates were comparable within the immune and nonimmune etiology (43\% vs. $53 \%$, $\mathrm{p}>0.05$ ). Of the 34 nonimmune hydrops fetalis (NIHF) cases, a plausible cause could be found for 24 (70.5\%). Post-mortem examinations were performed in 11 cases of 16 non-surviving infants with NIHF. The two factors that were associated independently with mortality in the stepwise multiple logistic regression analyses were a low 5 minute Apgar score and the need for surfactant treatment.

Conclusion Despite the improvements in postnatal care mortality rates are high and treatment options are still limited. The prenatal and postnatal procedures to improve lung capacity and function are necessary to achieve favorable outcome.

\section{VERY LOW BIRTH WEIGHT INFANTS IN ADNAN MENDERES UNIVERSITY NEONATAL INTENSIVE CARE UNIT}

doi:10.1136/archdischild-2012-302724.0801

D Engür, D Çolak, B Çetinkaya Çakmak, S Oktay, M Kaynak Türkmen. Adnan Menderes University, Aydin, Turkey

Background we retrospectively assessed mortality and morbidity rates of very low birth weight infants followed in Adnan Menderes University Neonatal Intensive Care Unit (NICU).

Methods 73 newborns with birth weight lower than $1500 \mathrm{~g}$ and gestational age under 32 weeks followed between January 2009 and December 2011 were included. Demographic characteristics, perinatal events, postnatal complications, duration of mechanical ventilation, mortality rates, length of hospital stay were investigated.

Results Mean gestational age was $28.1 \pm 2.4$ (23-32) weeks, mean birth weight was $1116.8 \pm 247.6(600-1500) \mathrm{g}$, mean mother age was $27.1 \pm 6.2$ (17-41). Median duration of ventilation was $173.4 \pm 182.2$ (0-912) hours. Twenty \% of the cases died; with median gestational age of $26.3 \pm 3.3$ (23-32) weeks and median birth weight of $886 \pm 310$ (600-1450) g. Sixty-four \% of the babies had mechanical ventilation support. Incidence of PDA, NEC, ROP were 19\%, 13.6\%, 19\% respectively. Nineteen \% of the infants had intracranial hemorrhage. Six infants had neurological abnormalities at discharge.

Abstract 801 Table 1 Characteristics of the infants

\begin{tabular}{ll}
\hline & $\%$ \\
\hline male & 55 \\
vaginal delivery & 38 \\
Antenatal Steroid & 30 \\
Mechanical Ventilation & 64 \\
Patent Ductus Arteriosus & 19 \\
Necrotizing Enterocolitis & 13,6 \\
Retinopathy of prematurity & 19 \\
late neonatal sepsis & 23,7 \\
intracranial hemorrhage & 19 \\
\hline
\end{tabular}

Conclusion Overall survival rates of our unit were found to be similar with previously reported rates from our country. Gestational age and birth weight correlated with survival and morbidity rates. Assessing outcomes of our NICU will provide us not only a new approach on understanding and management of these infants but also bring a perspective on predicting the prognosis and informing parents.

\section{CAUSES AND CONTRIBUTING FACTORS LEADING TO EXCHANGE TRANSFUSION :10 YEAR EXPERIENCE AT ADNAN MENDERES UNIVERSITY NEONATAL INTENSIVE CARE UNIT}

doi:10.1136/archdischild-2012-302724.0802

M Kaynak Türkmen, D Engür, D Çolak, S Oktay, A Aydoğdu, A Tosun. Adnan Menderes University, Aydin, Turkey

Background and Aims Jaundice is one of the most common problems in newborn period. Infants at risk for severe hyperbilirubinemia should be identified and closely monitored in order to avoid kernicterus. This study was performed to identify the etiology and sociodemographic characteristics of newborns with severe indirect hyperbilirubinemia who had exchange transfusion.

Methods The study sample included all infants who were undergone exchange transfusion at Adnan Menderes University NICU from January 2000 through December 2010. Hospital records were reviewed. Blood groups, direct Coombs test, whole blood count, peripheric blood smear, CRP, reticulocyte count, total, direct bilirubine levels, Tandem mass, G6PD and pyruvate kinase levels, urinalysis and urine culture, reducing substance in urine were investigated.

Results Sixty-five of the patients were male, 26.5\% preterm. Mean gestational age was $39.3 \pm 1.4$ for term infants, $35 \pm 1.1$ weeks for preterms. Mean serum total bilirubin level was $25 \pm 6.3 \mathrm{mg} / \mathrm{dl}$. Twentyfour \% of the patients had $\mathrm{Rh}$ incompatibility, \%24.5 had $\mathrm{ABO}$ incompatibility, $6.1 \%$ had both $\mathrm{Rh}$ and $\mathrm{ABO}$ incompatibility,\%4.1 G-6-PD deficient, 12.2\% had early breast milk jaundice, $\% 12.2$ had sepsis, $6.1 \%$ had hemolysis of unknown etiology. In five babies no etiology was discovered.

Conclusions Most of the infants had $\mathrm{Rh}$ or $\mathrm{ABO}$ incompatibility, who were vaginally delivered, discharged within 24 hours of birth and exclusively breastfed. Since mean age at admission was $3.4 \pm 3.7$ days, serum total bilirubin levels should be measured before discharge and discharge should be delayed in high risk babies.

\section{EFFECT OF BODY WEIGHT ON THE OUTCOME OF VENTRICULAR SEPTAL DEFECT REPAIR}

doi:10.1136/archdischild-2012-302724.0803

SR Ismail, A Dughiem, R Abusuliman, M Kabbani, H Najm. Cardiac Science, King Abdulaziz Medical City for National Guards, Pediatric Cardiac Intensive Care, Riyadh, Saudi Arabia

Introduction Low body weight and failure to thrive (FTT) often are considered limiting factors for open heart surgery. The purpose of our study is to asses the impact of FTT on ICU outcome of children undergoing Ventricular septal defect repair.

Methods A retrospective Study including all children less than 2 years who had VSD closure by open heart surgery during the period 2002-2010. Children were divided into 2 groups based on their weight for age using standard growth charts. Those with normal or mild failure to thrive ( $Z$ score $>-3$ ) are labeled as group A. group B included all children with severe failure to thrive $(Z$ score $\leq-3)$. Both groups were compared in term of all operative and early postoperative outcome parameters.

Results 145 patients were included. 58 cases in group A and 87 patients in group $\mathrm{B}$. The age and weight in group A was $(8 \pm 5.16$ 